\title{
Highlights of the 6th World Congress in Paediatric Cardiology and Cardiac Surgery
}

Nicholas Hayes', Henry Chubb', Srinivas Narayan' \& Shakeel Qureshi*1

'Evelina Children's Hospital, Guy's \& St Thomas' NHS Foundation Trust, Westminster Bridge Road, London, SET 7EH, UK

*Author for correspondence: Tel. : +44 2071884547 = Fax: +44 2071884556 w shakeel.qureshi@gstt.nhs.uk

\section{The World Congress in Paediatric Cardiology and Cardiac Surgery} Cape Town, South Africa, 17-22 February 2013

The 6th World Congress in Paediatric Cardiology and Cardiac Surgery took place in Cape Town, South Africa, in February 2013. The congress is the largest meeting in the field of congenital and paediatric heart disease and attracts a global audience of specialists with the aim of sharing the latest multidisciplinary developments in research and clinical practice. The congress was commended as a huge success and this article aims to give a general flavor of the diverse meeting through detailing a few specific highlights from the various tracks.

Held on 17-22 February 2013 in Cape Town, South Africa, The World Congress in Paediatric Cardiology and Cardiac Surgery represents the largest and one of the most important scientific meetings within the field of congenital heart disease. Meetings have taken place approximately once every 4 years since the first congress in 1980 and attract a global audience of specialists to highlight the latest developments and facilitate global collaboration of ideas. The meeting ran six simultaneous tracks:

- Surgery, anesthesia and intensive care;

- Catheter interventions from fetus to adult;

- Health systems and heart disease;

- Adults with congenital heart disease and the prevention of acquired heart disease starting in childhood;

- Cardiology and the imaging revolution;

- Nursing science.

With such a wide-ranging programme it is not feasible to document a comprehensive review of the entire meeting, but specific highlights have been detailed below.

\section{Catheter interventional track overview}

Departing from the usual World Congress programming, sessions on 3 days of the track included live case demonstrations from Frankfurt and Milan, as well as local centers from South Africa, which was a wonderful success. A large variety of cases were shown and there was plenty of interaction between the operators and the audience, which turned this into a highly educational event. Between the cases, there were lectures given by prominent international faculty members too. One of the major highlight lectures in the intervention track was a lecture by Elchanan Bruckheimer (Schneider Children's Medical Center Israel Petach Tikva, Israel) on a virtual catheter laboratory. He presented a futuristic view of how holography of the heart, based on newly developing technology, is likely to lead to interactive planning and performing of the interventions. There were exciting lectures highlighting recent and future developments. These included the development of new stents, fetal interventions in the future, biodegradable materials for pediatric cardiac interventions, the use of rotational angiography and the use of MRIguided cardiac catheterization and interventions. There was an interesting discussion in a lecture by John Thomson (Leeds General Infirmary, Leeds, UK) on whether the occlusion of patent foramen ovale was industry-driven or not as a result of forceful marketing strategies and whether there was sufficient data from studies to support device closure of patent foramen ovale. Some other new techniques were discussed, which included the early experience with percutaneous closure of the patent arterial duct in low birth weight infants (Neil Wilson; Oxford University Hospitals, Oxford, UK), stenting of the arterial duct in congenital heart disease (Mazeni Alwi; Institute Jantung Negara, Kuala Lumpur, Malaysia) and an update of the results of fetal aortic valvoplasty (Wayne Tworetzky; Boston Children's Hospital, MA, USA). There were some key lectures on improving the outcomes of interventions by developing interventional registries (Ziyad Hijazi; Rush University Medical Center, IL, USA),

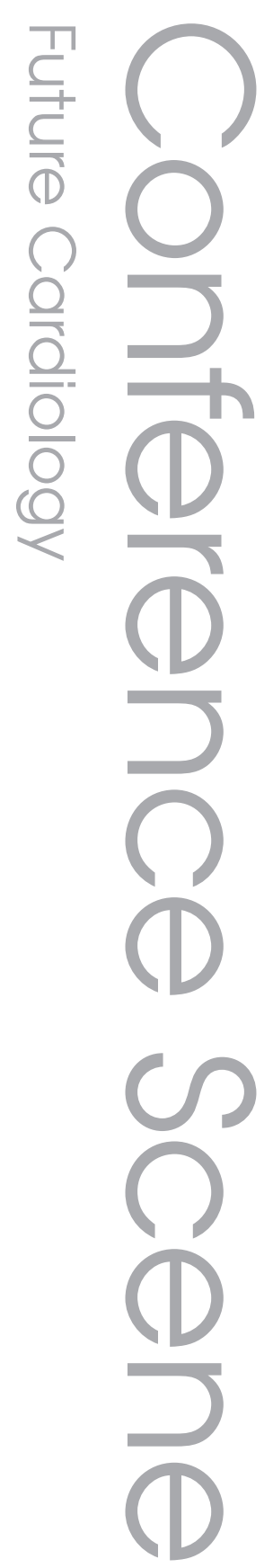

Future Medicine 
results of catheter closure of ventricular septal defects (Mario Carminati; San Donato Hospital, Milan, Italy), the very important topic of coarctation treatment by intervention from neonates to adults (Martin Schneider; Asklepios Klinik, Sankt Augustin, Germany) and the lessons that can be learnt from reporting adverse events after interventions (John Thomson). The latter was stimulating in trying to promote learning from the mistakes made by the operators themselves or others.

\section{Update on international results for the hybrid procedure for hypoplastic left heart syndrome: John Cheatham (Nationwide Children's Hospital, OH, USA)}

Over the past decade, the hybrid procedure for hypoplastic left heart syndrome has received a renewed degree of interest. Bilateral pulmonary artery banding and ductal stenting avoid the need for extensive arch repair and cardiopulmonary bypass in the immediate neonatal period. Since 2005, over 400 hybrid procedures have been performed in major centers in the USA, Canada, the UK, Brazil, Germany and Japan. Actuarial 1-year survival is approximately $70-80 \%$, although there is a significant learning curve with initial procedural mortality figures as high as $66 \%$ in the initial period and less optimal outcomes in units where the hybrid procedure is only performed in the highrisk cases (e.g., $<2.5 \mathrm{~kg}$ weight). Retrograde aortic arch obstruction occurs in around $20 \%$ of cases and either requires stenting or arch repair if the patient is old enough. The Hospital for Sick Children (Toronto, Canada) has advocated a reverse Blalock-Taussig shunt to supply the ascending aorta at the time of the initial hybrid procedure and the Evelina Children's Hospital (London, UK) have recently moved to a strategy of performing a standard Norwood procedure 1 at 6 weeks.

Data comparing neurodevelopmental outcomes, one of the main potential theoretical advantages of the hybrid procedure, are only just starting to emerge. Knirsch et al. analysed neuromotor scores at 3 and 12 months in 31 patients (Hybrid 13, NW 18) [1]. Neurodevelopmental outcome was significantly lower overall in patients with hypoplastic left heart syndrome compared with normal, with no significant difference between Norwood 1 and hybrid groups. However, this is a relatively small study and ongoing larger trials are required.

In conclusion, the results of hybrid therapy are comparable with conventional surgery and results are reproducible, but a learning curve exists, and treating retrograde arch obstruction remains a challenge. Longer-term assessment of pulmonary artery growth needs to be carried out and a prospective randomized study is currently underway.

\section{Treating hypertension by intervention: Horst Sievert (Sankt Katharinen- Krankenhaus, Frankfurt, Germany)}

Surgical thoracolumbar sympathectomy was effective at reducing blood pressure, but went out of fashion due to significant adverse effects. A similar reduction in blood pressure can be achieved by radiofrequency ablation of the sympathetic renal afferent nerves using radiofrequency catheters. The reduction in blood pressure can be sustained for up to 3 years and is most effective in patients with treatmentresistant essential hypertension, but can also be beneficial in patients with diabetes, heart failure and other diseases.

\section{Re-establishing vascular patency in the catheter laboratory: Henri Justino (Texas Children's Hospital, USA)}

The loss of venous patency occurs particularly in premature infants around peripherally inserted central catheter lines and the key learning point was the principle of not removing the offending line. Their team has found that the line itself is often the only route to allow passage of the wire through the occlusion, enabling removal of the clot with an endovascular clot removal catheter, often with very promising results. In larger patients, more aggressive approaches have often had to be adopted. Radiofrequency perforation through the occluded segment with multiple cutting balloon dilatations may be required and the boundaries of the territories of the interventional radiologists and cardiologists begin to be blurred. In a final case, Dr Justino described the complete reconstruction of the ileal/inferior vena cava system in a young adult with severe thrombophilia using multiple stents from the level of the hepatic veins down to the bifurcation of the ileal vessels. The results looked impressive, but the long-term outlook for such an approach remains to be seen.

\section{The failing right ventricle: Andrew Redington (The Hospital for Sick Children)}

The right ventricle (RV) is around four- to fivetimes more sensitive to afterload compared with the left ventricle (LV) and therefore the primary 
therapy to assist a failing RV should be focused on reducing the hydraulic impedance it faces (e.g., with pulmonary vasodilators). New concepts, however, are also focusing on the significant ventriculo-ventricular interaction. LV contraction contributes to $45 \%$ of RV contraction independently and, therefore, in acute isolated $\mathrm{RV}$ systolic failure, systemic vasoconstriction can increase RV output through effects on the LV. Furthermore, RV failure results in increased duration of RV systole and reduced diastolic LV filling. This pathophysiological process is aggravated by tachycardia and, consequently, rate control may be important in patients with pulmonary hypertension. $\beta$-blockers have traditionally been contraindicated in such patients, but ivabradine (which reduces heart rate without adversely affecting contractility) may have a specific role. The presence of RV fibrosis is a bad prognostic sign, but it may be possible to modify myocardial fibrosis using medications that affect endothelin receptors and TGF- $\beta$ (e.g., losar$\tan )$. Finally, it is interesting that patients with Eisenmenger's syndrome, despite having higher pulmonary vascular resistance, demonstrate better 5-year survival than patients with idiopathic pulmonary hypertension. A Potts shunt has therefore been performed in a few children with severe idiopathic pulmonary hypertension, resulting in prolonged survival and persistent improvement in functional capacities [2].

\section{Right ventricular function using conventional \& 3D echocardiography: Ben Eidem (Mayo Clinic, MN, USA) \& Tom Gentles (Green Lane \& Starship Children's Hospital, Auckland, New Zealand)}

The challenge with correct estimation of RV function is due to the geometry, anatomic location, beat to beat changes and pulmonary versus systemic vascular bed characteristics.

A segmental approach for the estimation of RV function involves estimation of the RV systolic function (including RV fractional area change, ejection fraction and $\mathrm{dP} / \mathrm{dt}$ ) and diastolic function (evaluated with tricuspid inflow Doppler echocardiography, hepatic venous Doppler and tissue Doppler).

TAPSE estimates the movement of tricuspid annulus during cardiac condition. It is quantitative, estimates longitudinal function and correlates with clinical outcome, particularly in pulmonary hypertension. It tends to increase with age and $Z$ scores for the TAPSE are available [3].

The biplane Simpson's method of fractional volume change has many limitations mainly, in geometric reconstruction, difficulty in imaging the RV free wall and inclusion of the right ventricular outflow tract.

$3 \mathrm{D}$ echocardiography can be used to estimate end diastolic and systolic volumes, and estimate the stroke volume and ejection fraction. All 3D echocardiography methods underestimated the end diastolic volume compared with MRI and the magnitude of underestimation increased with increasing RV size. Interobserver variability with 3D echocardiography is twice that of MRI.

\section{Emerging concepts for causes of congenital heart disease \& potential targets for remedial therapy}

Whilst prenatal environmental factors are responsible for a proportion of congenital heart disease, recent advances in genetic analysis have started to highlight the predominant genetic influence on the etiology of the majority of congenital heart disease. Signaling mutations associated with congenital heart disease are frequently expressed in the embryonic progenitors of cardiac fibroblasts and it appears that the fibroblast gene mutations are more important than mutations within the cardiac myocytes themselves. Lineages from cardiac fibroblasts are critical in developing the cardiac skeleton, valvuloseptal morphogenesis, the atrioventricular junction and control of myocyte proliferation. Target genes are typically downstream from transcription factors (e.g., TGF- $\beta$ ) and include the extracellular matrix signalling ligands, nodal kinases and GTPases. Examples of fibroblast mutations include the dachsous gene mutation, which results in marked myocyte hyperplasia and filamin A, in which mitral valve prolapse is observed. The mammalian heart also appears to have a postnatal window for regrowth/regeneration. CD $45^{+}$circulating progenitor cells continue to engraft the developing heart for a period of time following delivery.

Remedial therapy should therefore potentially be more focused on fibroblast mutations. Vectors used to inhibit abnormal signaling proteins, to transduce wild-type genes, to off-set mutations and to manipulate fibrosis/reactivate the innate ability of cardiac regeneration seen in the early postnatal period are all potential strategies to investigate in the future.

\section{Cell \& gene therapies for cardiac arrhythmias: Lior Gepstein (Rappaport Faculty of Medicine, Haifa, Israel)}

Professor Gepstein gave a whistle-stop tour of the advances in cell and gene therapies for cardiac arrhythmias. The field has made great advances 
over the last 10 years and is working towards human trials in a range of areas, representing potential therapies for tachyarrhythmias and bradyarrhythmias.

The goal for many groups has been the creation of a biological pacemaker. New technologies have enabled the creation of induced pluripotent cells, derived from fibroblasts, and these may be used to create pacemaker cells. These are capable, not only of generating a spontaneous rhythm, but are also sensitive to a range of endogenous signals to enable a degree of rate responsiveness. However, the other significant hurdle that needs to be overcome is that of conduction and engineered cells with induction of the expression of $\mathrm{Na}$ channels have shown promise in animal models, and optogenetics - light responsive channels, derived from algae - offer the potential for cardiac resynchronization. The question remains regarding the longevity of these cellular therapies, particularly in comparison with the level of functioning now achieved with electronic pacemakers. It may well be, though, that the greatest advantages for these new technologies are seen in children and pediatric cardiology may be the environment for longer-term introduction.

Professor Gepstein also touched on cellular therapies for tachyarrhythmias. Induction of potassium channels within myocardial cells extends the refractory period and early trials have shown inhibition of ventricular tachycardias with a ring of 'protecting' cells.

\section{The International Society for Nomenclature of Paediatric \& Congenital Heart Disease: the 4th International Summit}

The importance of a standardized and comprehensive internationally agreed nomenclature system in congenital heart disease in order to facilitate data collection, international collaboration, validation and benchmarking, cannot be overstated. Major progress has been made since the International Paediatric \& Congenital Cardiac Code was first established in 2005. The two previously established databases: European Paediatric Cardiac Code and Congenital Heart Surgery Nomenclature and Database Project have been combined and the number of agreed terms has increased from under 6000 in 2002 to over 17,000 in 2013 and continues to increase. Each definition assigned a unique six-digit code based on a hierarchical subset menu and a virtual museum of images is also being developed to try to assist with defining structural concepts. Recent developments include the incorporation of the entire International Paediatric and Congenital Cardiac Code subset into the Systemized Nomenclature of Medical Clinical Terms and the definitions of 311 higher-level terms are to be included in the beta draft of ICD-11 by WHO. The database is available free of charge at [101] and cross-mapping with local databases is encouraged to facilitate international collaboration and comparison.

Overall, the programme for this World Congress was highly varied and of interest to all participants, with stimulating sessions and takehome messages for the majority of attendees. The congress was also truly a world congress in that it was inclusive in the programme development, as well as in the involvement of speakers and participants from all continents.

Financial \& competing interests disclosure $S$ Qureshi has a consultancy to Numed Inc. (NY, USA) for Medtronic Inc. The authors have no other relevant affliations or financial involvement with any organization or entity with a financial interest in or financial conflict with the subject matter or materials discussed in the manuscript apart from those disclosed.

No writing assistance was utilized in the production of this manuscript.

\section{References}

1. Knirsch W, Liamlahi R, Hug MI et al. Mortality and neurodevelopmental outcome at 1 year of age comparing hybrid and Norwood procedures. Eur. J. Cardiothorac. Surg. 42(1), 33-39 (2012).

2. Baruteau AE, Serraf A, Lévy M et al. Potts shunt in children with idiopathic pulmonary arterial hypertension: long-term results. Ann Thorac. Surg. 94(3), 817-824 (2012).

3. Koestenberger M, Ravekes W, Everett AD et al. Right ventricular function in infants, children and adolescents: reference values of the tricuspid annular plane systolic excursion (TAPSE) in 640 healthy patients and calculation of z score values. J. Am. Soc Echocardiogr. 22(6), 715-719 (2009).

\author{
Website \\ 101. International Paediatric and Congenital \\ Cardiac Code. \\ www.ipccc.net
}

